\author{
Diese Stellungnahme wurde \\ ausgearbeitet von: \\ B. Hauer ${ }^{1}$ (federführend) \\ H. Mauch ${ }^{2}$ (federführend) \\ R. Loddenkemper ${ }^{1}$ (federführend), \\ unter Mitarbeit von \\ (in alphabetischer Reihenfolge). \\ M. Forßbohm ${ }^{3}$, J. Früh ${ }^{4}$, G. Loytved ${ }^{5}$, \\ K. Magdorf ${ }^{6}$, M. Priwitzer ${ }^{7}$, \\ S. Rüsch-Gerdes ${ }^{8}$, T. Schaberg ${ }^{9}$
}

\section{Der Tuberkulose-Schnelltest von DiaVita - keine Alternative zum Tuberkulinhauttest}

\author{
The Tuberculosis Rapid Assay from DiaVita - No Alternative to the \\ Tuberculin Skin Test
}

\section{Zusammenfassung}

In letzter Zeit werden auch auf dem deutschen Markt zunehmend neue Methoden zur Diagnostik einer behandlungsbedürftigen Tuberkulose und/oder einer latenten tuberkulösen Infektion angeboten. Bei dem von der Firma DiaVita vertriebenen Tuberkulose Schnelltest (TB-ST) handelt es sich um ein serologisches Testverfahren, welches bei an Tuberkulose erkrankten Patienten innerhalb weniger Minuten tuberkulose-spezifische Antikörper im Vollblut bzw. Serum nachweisen soll. Eine wissenschaftlich fundierte Bewertung des Tests ist aufgrund der eingeschränkten Datenlage kaum möglich. Bei von der Firma DiaVita mitgeteilter verhältnismäßig hoher Spezifität (über 95\%), aber unzureichender Sensitivität (ca. 60-80\%), insbesondere bei bakterienarmen Formen, ist der Test in der Routine nach unserer Einschätzung nur sehr eingeschränkt hilfreich. Der gezielte Einsatz bei dringendem Verdacht auf das Vorliegen einer behandlungsbedürftigen Tuberkulose kann bei positivem Ausfall den Verdacht erhärten, ein negatives Testresultat - aufgrund der zu erwartenden hohen Anzahl falsch negativer Ergebnisse - lässt hingegen keine verlässlichen differenzialdiagnostischen Rückschlüsse zu. Die höchste Aussagekraft besitzt der Test offensichtlich bei bakteriologisch bestätigten Tuberkulosen, wobei hier die Notwendigkeit einer ergänzenden Diagnostik hinterfragt werden muss. Für die Detektion einer latenten tuberkulösen Infektion ist der Test nicht evaluiert und nach unserer Einschätzung vo-

\section{Abstract}

New methods for diagnosing active tuberculosis (TB) and/or latent tuberculosis infection have been offered increasingly during recent years, also in Germany. The Tuberculosis Rapid Assay ('Tuberkulose Schnelltest [TB-ST]') from DiaVita is a serologic test method aiming to detect, within a few minutes, tuberculosisspecific antibodies in whole blood or serum of tuberculosis patients. Due to a lack of data, it is, however, hardly possible to assess this test on a scientific basis. With a relatively high specificity (according to DiaVita over 95\%) but inadequate sensitivity (approximately 60-80\%), particularly in paucibacillary types of $\mathrm{TB}$, we consider this method of limited value for routine testing. If active TB is strongly suspected, a positive test result may corroborate the tentative diagnosis, whereas a negative test result is not helpful for differential diagnosis considering the great number of false-negative test results to be expected. The greatest value of the test seems to be in bacteriologically confirmed TB, in which however there may be no need for additional diagnostics. The test has not been evaluated, and in our opinion is probably unsuitable for detecting latent TB infection. Data is also lacking for a number of patient groups in urgent need of a reliable alternative to the tuberculin skin test (e.g., immunocompromised patients, children). It is not clear whether this method will find a place in routine TB diagnostics. We therefore cannot recommend this test as a routine diagnostic tool for TB at present.

${ }^{4}$ Gesundheitsamt Waldshut

${ }^{5}$ Gesundheitsamt Würzburg

${ }^{6}$ Klinik für Pädiatrische Pneumologie, Lungenklinik Heckeshorn, Berlin

${ }^{7}$ Gesundheitsamt Stuttgart

${ }^{8}$ Nationales Referenzzentrum für Mykobakterien, Borstel ${ }^{9}$ Zentrum für Pneumologie, Diakoniekrankenhaus Rotenburg

Korrespondenzadresse Prof. Dr. med. Robert Loddenkemper · Deutsches Zentralkomitee zur Bekämpfung der Tuberkulose · Lungenklinik Heckeshorn·Zum Heckeshorn 33 · 14109 Berlin·E-mail: loddheck@zedat.fu-berlin.de 
raussichtlich ungeeignet. Darüber hinaus fehlen Daten zur Anwendung bei vielen Patientengruppen, bei denen eine zuverlässige Alternative zum Tuberkulinhauttest besonders dringlich wäre (z.B. immunsupprimierte Patienten, Kinder). Ob die Methode einen Platz in der Routinediagnostik der Tuberkulose finden wird, ist derzeit nicht beurteilbar. Zum jetzigen Zeitpunkt kann der routinemäßige Einsatz des Tests in der Tuberkulosediagnostik nicht empfohlen werden.

\section{Hintergrund}

Schon die Umstellung von Stempeltestverfahren auf die intrakutane Tuberkulinapplikation nach Mendel-Mantoux hat bei vielen mit Tuberkulosekontrolle und -diagnostik befassten Kolleginnen und Kollegen zu großer Verunsicherung geführt. Diese Situation wurde durch den Produktionsstopp des bisher in Deutschland verwendeten Tuberkulins GT Behring und dadurch zeitweise nur nach § 73 Arzneimittelgesetz möglichen Import ausländisch produzierten Tuberkulins verschärft [6]. Durch diese Entwicklungen wurde der Wunsch nach einer vernünftigen Alternative zum Tuberkulinhauttest laut, zumal dieser bekanntlich, ebenso wie die übrigen zur Verfügung stehenden klassischen Diagnoseverfahren, nicht über eine optimale Sensitivität und Spezifität verfügt.

Serologische Testverfahren (humorale Immunität) zur Detektion Tuberkulose-spezifischer Antikörper werden weltweit seit über 15 Jahren entwickelt [2 -8]. Zum Einsatz kommen Tuberkulosespezifische Antigene wie beispielsweise das 38, 23/24, 19, 17 oder das 16-(14) kDa-Antigen, sowie Mtb8, ESAT-6 und A60. Sie werden entweder isoliert oder als Antigen-,,Cocktail“ verwendet [9-11]. Das $38 \mathrm{kDa}$-Antigen erzielt im Vergleich zu den anderen Antigenen offensichtlich die höchste Spezifität und Sensitivität bei aktiver Erkrankung $[3,4,12,13]$. Dagegen wird angenommen, dass eine Immunantwort auf beispielsweise das $14 \mathrm{kDA}$ - und ESAT-6-Antigen möglicherweise als Hinweis auf eine latente tuberkulöse Infektion gedeutet werden kann [9]. Neben der ELISATechnik (als quantitativer Test) stehen mittlerweile auch Schnelltestverfahren (als qualitativer Test) zur Verfügung $[8,9,11,14-16]$.

$\mathrm{Zu}$ den letztgenannten Schnelltestverfahren zählt auch der von der DiaVita GmbH/Heidelberg vertriebene serologische Tuberkulose-Schnelltest (TB-ST), welchen die Firma alternativ zum Tuberkulinhauttest als diagnostisches Mittel zur raschen Detektion einer behandlungsbedürftigen Tuberkulose anbietet. In der Informationsbroschüre [14] wird der TB-ST als „,idealer Test sowohl für Einzel- als auch für Reihenuntersuchungen“ angeboten. Mit seiner Hilfe sei „eine schnelle und sichere Aussage über das Vorliegen einer aktiven Tuberkulose möglich“. Das Testergebnis soll weder durch eine stattgefundene BCG-Impfung noch durch eine „inaktive“ Infektion oder Infektionen mit anderen Mykobakterien verfälscht werden. Die Sensitivität des Tests gibt die Firma mit $60-80 \%$, die Spezifität mit $>95 \%$ an.
Bislang konnten sich serologische Tests in der Tuberkulosediagnostik nicht etablieren. Dies liegt zum einen an den bisher nach wie vor nur ungenügend verstandenen immunologischen Prozessen, die sich nach der Infektion mit Tuberkulosebakterien abspielen, wie z. B. der Dynamik der Immunglobulinproduktion. So sind die Ursachen interindividueller Immunreaktionen und die Rolle, die hierbei der Schweregrad und die Lokalisation der Tuberkulose spielen, noch weitgehend unbekannt. Dies gilt auch für den Einfluss, den eine Behandlung mit Tuberkulosemedikamenten auf die Antikörperproduktion hat, sowie für die Auswirkungen bei Vorliegen einer Medikamentenresistenz $[4,18]$.

Zum anderen konnten die serologischen Testverfahren auch aufgrund ihrer unbefriedigend niedrigen und sehr variablen Sensitivitätsraten (20-80\%) bislang nicht überzeugen $[2,9,11,15]$. Insbesondere der Schweregrad der Tuberkulose wirkt sich auf die Sensitivität der Tests aus. So liegt diese für im Sputum mikroskopisch positive Patienten in der Regel bei $60-80 \%$, sinkt aber für sputum-negative bereits auf unter $50 \%$ ab und erreicht für extrapulmonale (und damit in aller Regel bakterienarme) Tuberkuloseformen höchst unbefriedigende Sensitivitäten um 20-30\% $[3,9]$. Auch im Kindesalter werden nur geringe Sensitivitäten erreicht $[3,19]$. Ebenso wurden bei HIV-positiven Personen deutlich geringere Sensitivitäten beobachtet $[3,11,20]$.

Die Spezifität der serologischen Testverfahren bewegt sich, je nach verwendetem Antigen, meist um $90 \%$ und teilweise auch darüber. Leider wird sie oft zu hoch eingeschätzt, weil die Untersuchungen meist an einem überwiegend gesunden Studienkollektiv durchgeführt werden.

Bis dato bestehen für wichtige Fragestellungen noch große Informationslücken, so beispielsweise zum Einfluss des Immunstatus des Patienten auf das Testresultat (HIV, s.o.; immunsuppressiv Behandelte) oder zum Verlauf der humoralen Antwort und damit der Testreaktion unter antituberkulotischer Therapie. Ungeklärt bzw. zweifelhaft ist das Vermögen serologischer Tests, zuverlässig zwischen aktiver Tuberkulose, ausgeheilter Tuberkulose und latenter Infektion zu unterscheiden.

Ungenügend evaluiert sind auch Faktoren wie der störende Einfluss durch Umweltmykobakterien (Synonyme: nichttuberkulöse, atypische, ubiquitäre Mykobakterien), die Auswirkung regionaler Häufungen von genetisch unterschiedlichen Mycobacterium tuberculosis-Stämmen mit unterschiedlichem AntigenSpektrum und die Bedeutung des HLA-DR Phänotyps des Getesteten [3].

\section{Funktionsweise des Tuberkulose-Schnelltests}

Beim TB-ST handelt es sich um ein in-vitro-Testverfahren im Sinne eines immunochromatografischen Tests [14]. Die Testplatte enthält eine Membran, auf welcher antikörperbindende Proteine, welche an kolloidale Goldpartikel konjugiert sind, fixiert sind. Nach Aufbringen von wenigen Tropfen Vollblut oder Serum $(20 \mu \mathrm{l})$ in die Vertiefung einer Testplatte und Zugabe eines Puffers wandert die aufgetragene Flüssigkeit entlang der Membran. Dabei sollen die in der Probe enthaltenen TB-Antikörper - sofern vorhanden - bei Passage des antikörperbindenden Protein/Gold- 
komplex dort gebunden werden. Bei Vorhandensein von TB-Antikörpern entwickelt sich dann eine pink- bzw. purpurfarbene Bande im Testfeld. Bei weiterer Passage entsteht in einem Kontrollfeld ebenfalls ein Teststreifen, welcher, vergleichbar mit der Funktionsweise eines Schwangerschaftstests, anzeigt, ob der Test ordnungsgemäß ausgeführt wurde. Jede Linie, egal welcher Ausprägung und Stärke, die innerhalb von 25 Minuten im Testfeld erscheint, ist als positiver Befund zu werten. Bei negativer Reaktion im Kontrollfeld ist der Test zu wiederholen.

Als Antigene werden PstS1 (LIONEX-Patent, ein 38 kDa-Antigen) sowie geringe Mengen des $16 \mathrm{kDa}$-Antigens verwendet [persönliche Mitteilung Prof. M. Singh, 12.07.2005].

\section{Datenlage}

Der TB-ST wird bereits seit Anfang der 90er-Jahre von der Firma LIONEX in Braunschweig unter Leitung von Prof. Mahavir Singh entwickelt. Auf Anfrage erhielten wir vorläufige und bisher unveröffentlichte Studiendaten, bei welchen das Testverfahren an insgesamt 483 Personen in drei Studienzentren (Deutschland, USA und Indien) zum Einsatz kam. Davon litten 182 Personen an einer bakteriologisch bestätigten oder klinisch diagnostizierten pulmonalen Tuberkulose. Als Kontrollgruppe dienten 301 Personen, welche entweder gesund waren oder an anderen Infektionen litten. Die Spezifität in der Kontrollgruppe wurde mit 98,7\% errechnet (297/301 richtig negative Ergebnisse), die Sensitivität liegt zwischen $73 \%$ für Sputum-negative und $78 \%$ für bakteriologisch bestätigte Tuberkulosen.

Des Weiteren erhielten wir Einsicht in erste Auswertungen der in Deutschland erhobenen Daten an 88 bestätigten pulmonalen Tuberkulosen und einer Kontrollgruppe von 338 Personen (darunter gesunde und Patienten mit anderen, z.T. auch respiratorischen Erkrankungen). Die hier erreichte Spezifität betrug ca. 96\%, die Sensitivität lag bei 70\% [persönliche Mitteilung Prof. M. Singh, 17.07.2005].

Zusammenfassend lässt sich zum jetzigen Zeitpunkt aus den uns vorliegenden Daten folgende Einschätzung des aktuellen Stellenwertes des TB-ST in der Tuberkulosediagnostik abgeben:

Es liegen derzeit keine wissenschaftlichen Publikationen von Studienergebnissen zum TB-ST vor. Die von der Firma zur Verfügung gestellten Daten sind nach unserer Einschätzung für eine wissenschaftlich fundierte Beurteilung nicht ausreichend.

Bislang wurde der Test fast ausschließlich bei pulmonalen Tuberkulosen untersucht, eine Aussage zur Eignung des Tests bei extrapulmonalen Tuberkulosen ist damit nicht möglich.

Das Testverfahren wurde für die Diagnostik einer latenten tuberkulösen Infektion (LTBI) nicht evaluiert, er ist daher zum Einsatz bei Umgebungsuntersuchungen oder Tuberkulose-ScreeningUntersuchungen zur Detektion einer LTBI nicht zu empfehlen. Es ist zu vermuten, dass hier die Sensitivität noch deutlich unterhalb der mit serologischen Tests üblicherweise erreichten geringen Sensitivitäten bei geschlossenen und extrapulmonalen $\mathrm{Tu}-$ berkuloseformen liegt.
Es bleibt auch unklar, ob sich der Test zur Differenzierung zwischen einer aktiven Erkrankung und einer latenten Infektion mit M. tuberculosis eignet. Die Antigenzubereitung des TB-ST enthält überwiegend das $38 \mathrm{kDa}$ Antigen und in geringerer Form das $16 \mathrm{kDa}$ Antigen. Ersteres soll eine aktive Tuberkulose, letzteres eine latente tuberkulöse Infektion erkennen [persönl. Mitteilung Prof. Singh 17.07.2005]. Damit wäre eine eindeutige Unterscheidung zwischen aktiver Tuberkulose und latenter Tuberkuloseinfektion jedoch nicht möglich.

Die gewählte Kontrollgruppe setzt sich aus gesunden und an „anderen Infektionen“ erkrankten Personen zusammen. Ein diagnostischer Test muss sich aber vor allem bei den Zielgruppen bewähren, welche an differenzialdiagnostisch bedeutsamen Krankheitsbildern leiden bzw. bei denen bestimmte Gesundheitszustände das Ergebnis eines solchen Testes stören könnten $[5,19]$. Im Fall der Tuberkulose wären dies beispielsweise pneumologische Krankheitsbilder wie unspezifische Pneumonien, Erkrankungen durch Umweltmykobakterien, COPD und Sarkoidose. Berücksichtigt werden müssen darüber hinaus Zustände, die mit einem beeinträchtigten Immunsystem einhergehen, insbesondere HIV, Tumorleiden und iatrogene Immunsuppression (z.B. nach Organtransplantation). Nur eine hohe Spezifität auch in diesen Personengruppen würde die Häufigkeit falsch positiver Befunde und damit möglicher falscher Therapien minimieren.

Problematisch bei dem TB-ST ist vor allem die unzureichende Sensitivität, insbesondere bei bakterienarmen Tuberkuloseformen. Dabei wäre gerade bei den bakteriologisch nicht bestätigten Tuberkulosen ein zusätzliches diagnostisches Mittel sehr hilfreich.

\section{Fazit}

Zusammenfassend lässt sich sagen, dass bei Anwendung des TBST bei Verdacht auf eine behandlungsbedürftige Tuberkulose ein positives Testergebnis das Vorliegen einer aktiven Erkrankung wahrscheinlicher macht. Die abschließende Diagnose muss sich dabei selbstverständlich immer in erster Linie auf die herkömmlichen Befunde (Radiologie, Klinik, Bakteriologie, Tuberkulinhauttest) stützen. Die beste Aussagekraft hat der Test bei Patienten mit mikroskopisch positivem Sputumbefund, wobei es hier aber in aller Regel keines zusätzlichen diagnostischen Mittels bedarf und für hiesige klinische Belange annähernd genauso schnelle hochspezifische Bestätigungsverfahren wie die PCR mit erheblich besserer Sensitivität verfügbar sind. Beim Patienten mit bakterienarmer pulmonaler Tuberkulose sowie vermutlich auch bei extrapulmonalen Tuberkuloseformen (z. B. Meningitis tuberculosa) ist der Test als Bestätigungstest unzuverlässig, da mit einer hohen Anzahl falsch negativer Resultate gerechnet werden muss.

Im Falle eines negativen Testausfalles ist keine zusätzliche Information gewonnen, da die Wahrscheinlichkeit eines falsch negativen Testausfalls aufgrund der eher niedrigen Sensitivität hoch ist, insbesondere in den eben genannten Fällen. 
Wie bei jeder diagnostischen Methode hängen die prädiktiven Werte, d.h. die Wahrscheinlichkeiten, ob eine Person mit positivem Testergebnis auch wirklich erkrankt bzw. mit negativem Ergebnis auch wirklich gesund ist, von der Häufigkeit des Vorkommens einer Erkrankung, d.h. von der Erkrankungsprävalenz ab. In Populationen mit einer hohen Tuberkuloseprävalenz, wie z.B. in Indien oder Ländern des südlichen Afrikas, sind diese Werte deutlich höher als in einem Niedrigprävalenzland wie Deutschland. Daher ist bei in Hochprävalenzländern durchgeführten Studien die Übertragbarkeit der Ergebnisse auf andere epidemiologische Verhältnisse stets zu hinterfragen.

Größere und sorgfältig geplante Studien wären daher erforderlich, um den TB-ST wissenschaftlich fundiert hinsichtlich aller angesprochenen offenen Fragestellungen bewerten zu können und daraus mögliche konkrete Indikationen für ein TuberkuloseNiedrigprävalenzland wie Deutschland abzuleiten.

Insgesamt halten wir den Stellenwert des TB-ST zur Diagnostik einer behandlungsbedürftigen Tuberkulose zum jetzigen Zeitpunkt für marginal. Aufgrund der bisher vorliegenden Datenlage kann der Test auf keinen Fall zur Diagnostik einer latenten tuberkulösen Infektion bzw. als Screeningmethode empfohlen werden.

\section{Danksagung}

Das DZK bedankt sich für die finanzielle Unterstützung durch das Bundesministerium für Gesundheit und Soziale Sicherung, die auch diese Publikation ermöglicht hat.
Literatur

${ }^{1}$ Sagebiel D, Hauer B, Haas W et al. Zukünftige Tuberkulinversorgung in Deutschland. Bundesgesundheitsbl - Gesundheitsforsch - Gesundheitsschutz 2005; 48: 477-482

${ }^{2}$ Bothamley GH, Rudd R, Festenstein F et al. Clinical value of the measurement of Mycobacterium tuberculosis specific antibody in pulmonary tuberculosis. Thorax 1992; 47: 270-275

${ }^{3}$ Bothamley GH. Serological diagnosis of tuberculosis. Eur Respir J 1995; 8: 676s-688s

${ }^{4}$ Jackett PS, Bothamley GH, Batra HV et al. Specificity of antibodies to immunodominant mycobacterial antigens in pulmonary tuberculosis. J Clin Microbiol 1988; 26: 2313-2318

${ }^{5}$ Mauch H. Probleme der immunserologischen Diagnostik bei Infektionen durch Mykobakterien. Klin Lab 1992; 38: 342-344

${ }^{6}$ Sorensen AL, Nagai S, Hoen G et al. Publication and characterization of a low-molecular-mass T-cell antigen secreted by Mycobacterium tuberculosis. Infect Immun 1995; 63: 1710-1717

${ }^{7}$ Verbon A. Development of serological tests for tuberculosis - problems and potential. Trop Geograph Med 1994; 46: 275-279

8 Pottumarthy S, Wells VC, Moris AJ. A comparison of seven tests for serological diagnosis of tuberculosis. J Clin Microbiol 2000; 38: 2227-2231

${ }^{9}$ Amicosante M, Houde M, Guaraldi G. Sensitivity and specificity of a multi-antigen ELISA test for the serological diagnosis of tuberculosis. Int J Tuberc Lung Dis 1999; 3: 736-740

${ }^{10}$ Lyashchenko KP, Singh M, Colangeli R et al. A multi-antigen print immunoassay for the development of serological diagnosis of infectious diseases. J Immunol Meth 2000; 242: 91 - 100

11 Perkins MD, Conde MB, Martins M et al. Serological diagnosis of tuberculosis using a simple commercial multiantigen assay. Chest 2003; 123: $107-112$

12 Silva VMC, Kanujia G, Gennaro ML et al. Factors associated with humoral response to ESAT-6, $38 \mathrm{kDa}$ and $14 \mathrm{kDa}$ in patients with a spectrum of tuberculosis. Int J Tuberc Lung Dis 2003; 7: 478-484

${ }^{13}$ Wilkinson RJ, Hasløv K, Rappuoli R et al. Evaluation of the recombinant 38-Kilodalton antigen of Mycobacterium tuberculosis as a potential immunodiagnostic reagent. J Clin Microbiol 1997; 35: 553-337

${ }^{14}$ DiaVita. TB-ST Tuberkulose-Schnelltest. Der schnellste Tuberkulosetest aller Zeit!

${ }^{15}$ Grobusch MP, Schürmann D, Schwenke S et al. Letters to the Editor. Rapid Immunochromatographic Assay for Diagnosis of Tuberculosis. J Clin Microbiol 1998; 36: 3443

${ }^{16}$ Bartoloni A, Strohmeyer M, Bartalesi F et al. Evaluation of a rapid immunochromatographic test for the serologic diagnosis of tuberculosis in Italy. CMI 2003; 9: 632 - 639

${ }^{17}$ Lyashchenko K, Colangeli R, Houde M et al. Heterogeneous antibody responses in tuberculosis. Infect Immun 1998; 66: 3936-3940

18 Bothamley GH. Epitope-specific antibody levels demonstrate recognition of new epitopes and changes in titer but not affinity during treatment of tuberculosis. Clin Diagn Lab Immunol 2004; 22: 942 - 951

${ }^{19}$ Imaz MS, Comini MA, Zerbini E et al. Evaluation of the diagnostic value of measuring IgG, IgM and IgA antibodies to the recombinant 16-kilodalton antigen of Mycobacterium tuberculosis in childhood tuberculosis. Int J Tuberc Lung Dis 2001; 11: 1036-1043

${ }^{20}$ Mathur ML, LoBue PA, Catanzaro A. Evaluation of a serological test for the diagnosis of tuberculosis. Int J Tuberc Lung Dis (1999); 3: $732-735$ 